\title{
Uma Estratégia Regulatória Local para a Educação Básica no Rio de Janeiro
}

\author{
Carlos Emmanuel Joppert Ragazzo' \\ Guilherme da Franca Couto Fernandes de Almeida' \\ 'Fundação Getúlio Vargas (FGV), Rio de Janeiro/RJ - Brasil
}

RESUMO - Uma Estratégia Regulatória Local para a Educação Básica no Rio de Janeiro ${ }^{1}$. Este artigo explora dados referentes ao desempenho das escolas municipais do Rio de Janeiro nos primeiros anos do Ensino Fundamental para apurar que medidas podem ser utilizadas para complementar os instrumentos regulatórios top down voltados para a educação, evidenciando que fatores locais exercem influência expressiva sobre a qualidade do ensino. Na cidade, escolas vizinhas apresentam resultados diametralmente opostos, apesar de serem comparáveis em termos socioeconômicos. Essa realidade sugere que instrumentos regulatórios bottom-up podem ser usados de maneira eficiente. Há, porém, uma lacuna normativa nesse sentido, com a forte prevalência de políticas públicas top-down.

Palavras-chave: Educação Básica. Regulação bottom-up. Gestão Escolar.

ABSTRACT - A Local Regulatory Strategy for Basic Education in Rio de Janeiro. This paper explores data about the performance of municipal schools in Rio de Janeiro during the first years of basic education to show that the regulatory instruments currently employed by the federal government must be complemented by a strategy that focus on local factors. Using data about the geographical location of Rio de Janeiro municipal schools and their performance at IDEB, we were able to pick out neighboring schools that achieved diametrically opposed results, despite serving students with comparable socioeconomic status. This reality suggests that bottom-up regulatory instruments might be employed efficiently. There is, however, a normative gap in that regard, with a strong prevalence of top-down public policies currently in place.

Keywords: Basic education. Bottom-up regulation. School Administration.

Educação \& Realidade, Porto Alegre, v. 45, n. 3, e93964, 2020.

http://dx.doi.org/10.1590/2175-623693964 
Uma Estratégia Regulatória Local para a Educação Básica no Rio de Janeiro

\section{Introdução}

Há amplo consenso político e social a respeito da importância da educação básica pública. Prova disso são as repetidas menções a ela na $\mathrm{CRFB} / 88$ e a vinculação orçamentária dos recursos de cada ente federativo à educação, estabelecida por força do art. $212^{2}$. A decisão política de proteger constitucionalmente a educação básica pública foi fruto de uma mobilização da sociedade civil durante a constituinte que não deixa dúvidas sobre sua centralidade (Martins, 2018). Iniciativas recentes da sociedade civil, como o Todos pela Educação e o Mapa da Educação, mostram que o compromisso dos brasileiros com a pauta da educação básica não esmoreceu ao longo dos 30 anos que nos separam da constituinte.

As opções específicas do constituinte são informativas, ainda, com relação a outro aspecto importante do nosso argumento. Elas são sintomáticas do tipo de atenção que o Direito, por meio de seus instrumentos regulatórios, tem dispensado à educação: há um foco em instrumentos top-down ${ }^{3}$, como, por exemplo, programas de financiamento federais (e também de transferências federais para merenda, transporte e livros didáticos), definição de bases curriculares nacionais, programas de formação de professores, entre outros. Em larga medida, esse foco foi uma estratégia efetiva para melhorar educação de uma forma sensível: desde o início da mensuração do $\mathrm{IDEB}^{4}$, houve expressivo avanço no desempenho das escolas brasileiras ${ }^{5}$, demonstrando que as políticas públicas de valorização, investimento e mensuração do ensino básico (Coelho, 2008) foram, pelo menos em parte, bem-sucedidas.

Esse resultado faz sentido, já que a literatura especializada indica que fatores como a infraestrutura das escolas (vide Alves; Soares, 2013b), o nível socioeconômico dos alunos (Alves; Soares, 2007a) e outras variáveis sociais e demográficas (Andrade; Laros, 2007) exercem influência expressiva sobre o IDEB. De toda forma, resta muito a ser feito: apesar da adoção das políticas mencionadas, no último PISA, avaliação internacional de ensino, o desempenho do país ficou muito aquém do desejável (Sassaki et al., 2018).

Dado o atual cenário de crise e os avanços que já foram realizados por meio de políticas top-down, parece útil buscar estratégias diferentes e complementares para a educação básica pública. Fatores no nível da escola relacionados à gestão, às práticas pedagógicas, à autonomia (Bloom et al., 2015) e à integração com a comunidade (Cunha et al., 2017) exercem influência significativa sobre o desempenho dos alunos. Portanto, a gestão pública e, em especial, a regulação da educação, deve adotar estratégias complementares aos instrumentos centralizadores que caracterizaram o avanço da educação nos últimos anos.

A necessidade de um leque regulatório mais amplo também decorre da multiplicidade de situações que compõem o problema da educação no Brasil, já que existem diferentes estágios de desenvolvimento não apenas entre estados, como também entre municípios e, mais ainda, entre escolas que compõem a rede de um mesmo município. É 
razoável pensar que um determinado grau de infraestrutura (incluindo-se aí acesso a merenda, transporte e conteúdo escolar), um plano de formação para professores e uma base curricular sejam condições necessárias para que as escolas alcancem melhores resultados. Após certo ponto, porém, esses instrumentos passam a ter menor impacto nos resultados de aprendizagem, razão pela qual o custo de oportunidade das diferentes estratégias regulatórias possíveis deve ser levado em consideração, de forma a otimizar o processo de melhora dos indicadores de aprendizagem.

Para demonstrar a utilidade de estratégias complementares aos instrumentos regulatórios que já vêm sendo utilizados pelo Governo Federal Brasileiro, este artigo irá analisar se: (i) os resultados de aprendizagem entre diferentes escolas de uma mesma rede escolar de um município possuem diferença relevante entre si e (ii) se instrumentos regulatórios baseados numa estratégia bottom-up poderiam ser utilizados de forma complementar com o objetivo de melhorar os resultados da aprendizagem.

O município do Rio de Janeiro será utilizado como caso teste para as hipóteses acima. Este artigo irá utilizar dados georreferenciados do município (PCRJ, 2019) sobre a performance das escolas municipais no IDEB, com recorte específico nos primeiros anos do Ensino Fundamental (Fundamental I). Estes dados permitem verificar se existem escolas vizinhas - e, portanto, comparáveis - com resultados muito discrepantes. As informações colhidas pelo INEP e pela prefeitura do Rio permitem, ainda, testar se eventuais diferenças entre pares de escolas podem ser reduzidas a fatores socioeconômicos, medidos por meio do INSE $^{6} \mathrm{e}$ dos limites de favelas.

A proximidade física de pares discrepantes de escolas, se detectada, é indicativa de que pelo menos alguns dos desafios atrelados à melhoria na qualidade da educação básica precisam ser enfrentados a partir de instrumentos regulatórios complementares às medidas federais mencionadas, em especial sob a abordagem bottom-up. Afinal, caso seja comprovado que algumas escolas conseguem bons resultados (ou evoluções relevantes nos indicadores) apesar das dificuldades que enfrentam, as suas práticas podem servir de exemplo para instruir medidas de recuperação de unidades que apresentam pior desempenho. Esse aprendizado com as experiências locais é o que caracteriza as estratégias regulatórias bottom-up, restando verificar, ainda, a melhor forma de implementá-las (se por meio de auxílio por parte do Governo Federal ou se por meio de uma mudança na governança regulatória dos municípios, a fim de incorporar programas e/ou medidas regulatórias bottom-up em seu portfólio).

A opção pelos anos iniciais do Ensino Fundamental se justifica a partir do seu provável impacto nos segmentos posteriores (anos finais do Ensino Fundamental e Ensino Médio). A hipótese é de que as deficiências de ensino nos primeiros anos do Ensino Fundamental prejudicam o conteúdo que precisa ser apreendido pelo aluno nas séries finais e gera consequências até o Ensino Médio7. As estatísticas são consis- 
Uma Estratégia Regulatória Local para a Educação Básica no Rio de Janeiro

tentes com essa hipótese. Ao se olhar apenas para o aprendizado em matemática, por exemplo, apenas $42,9 \%$ dos alunos saem do Ensino Fundamental I com um nível adequado de aprendizagem. Esse número desce para 18,2\% ao final do Ensino Fundamental II e 7,3\% ao final do ensino médio ${ }^{8}$ (Todos pela Educação, 2018). Presumivelmente, é mais difícil aprender para os alunos que saíram dos primeiros anos do Ensino Fundamental com uma habilidade insuficiente nas matérias centrais do currículo, o que pode gerar não só um efeito cascata na performance dos estudantes ao longo do tempo, com possíveis impactos em evasão escolar, em função do seu efeito na relação idade/aluno ano. A mesma história se repete para o desempenho em língua portuguesa, com dados similares. Se esse raciocínio possui algum mérito, a maneira mais eficiente de alocar recursos é sequencial, enfrentando primeiro os anos iniciais do ensino e, posteriormente, seus anos finais. A opção pelo município do Rio de Janeiro, por sua vez, foi mais pragmática: a cidade se destaca como centro de referência em dados abertos (Schreiner, 2016, p. 26).

\section{Diferenças em Resultados de Aprendizagem na Mesma Rede Escolar: possibilidade de uma abordagem bottom-up}

Existem fortes indicativos de que estratégias regulatórias bottom-up podem representar avanços significativos para a qualidade de aprendizagem: a pesquisa Excelência com Equidade (Faria; Guimarães, 2015; Fundação Lemann; Itaú BBA, 2012), que teve como objetivo identificar e pesquisar quantitativa e qualitativamente algumas escolas-referência nas diferentes regiões do Brasil, apontou para importância de bons diretores e boas práticas de gestão no nível da escola, chamando atenção para peculiaridades do ensino difíceis de regular por meio de instrumentos centralizadores. Quantitativamente, esses esforços fazem diferença: Bloom et al. (2015), em estudo que investigou oito países, incluindo o Brasil, mostraram o alto grau de influência exercido pelas práticas de gestão das escolas - em particular, seu grau de autonomia administrativa - sobre o desempenho acadêmico de seus alunos.

Levando em consideração o que foi dito acima a respeito do impacto de medidas específicas de gestão, a necessidade de instrumentos regulatórios complementares faz sentido se (i) existirem escolas municipais com desempenhos discrepantes no IDEB nos anos iniciais do ensino fundamental e (ii) essas escolas forem próximas em termos físicos e socioeconômicos. Usando dados da prefeitura do Rio de Janeiro (PCRJ, 2019) e os microdados do IDEB (INEP, 2020), foi possível explorar essas hipóteses, criando um mapa de calor onde cada ponto representa uma escola da rede municipal e a temperatura da cor representa o IDEB da escola. Quanto mais escura a cor, menor o IDEB da escola. 


\section{Figura 1 - IDEB no ensino básico das escolas municipais do Rio de} Janeiro $^{9}$

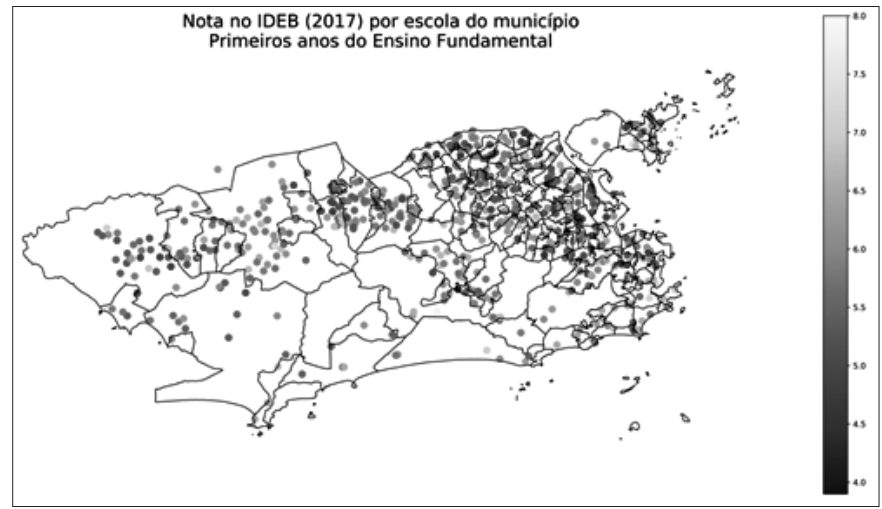

Fonte: elaboração própria, a partir dos dados do IDEB (referentes a 2017; INEP, 2020) e da prefeitura do Rio de Janeiro (PCRJ, 2019).

O mapa acima mostra que as duas hipóteses parecem plausíveis. De maneira consistente com a literatura especializada a respeito dos fatores que influenciam o desempenho escolar (Alves; Soares, 2007a, Alves; Soares, 2013b; Andrade; Laros, 2007; Gramani, 2017), algumas áreas da cidade possuem uma concentração maior de escolas com baixo desempenho, comprovando o peso de questões socioeconômicas no resultado de aprendizagem. No entanto, em praticamente todos os bairros da cidade do Rio de Janeiro, é possível observar pontos bem mais claros (IDEBs mais altos) ao lado de pontos mais escuros (IDEBs mais baixos), indicando que de fato existem escolas vizinhas com desempenho substancialmente diferente.

\section{Existência de Escolas-Referência e Escolas de Baixo Desempenho na Rede Escolar}

Quais escolas podem ser consideradas referência nesse contexto? Se o objetivo é encontrar as variáveis no nível da escola que podem ser manipuladas para fazer com que escolas com desempenho ruim alcancem um patamar aceitável de qualidade, o ideal é identificar as escolas que passaram por essa história. Assim, ao invés de focar única e exclusivamente no desempenho das escolas na última aferição do IDEB, se optou por selecionar as escolas que apresentaram uma evolução expressiva entre os IDEBs de 2005 e 2017. Foram criadas 2 categorias diferentes para as escolas-referência: 1) escolas que foram de desempenho muito ruim para desempenho bom: nessa primeira classificação estão as escolas que tiveram IDEB abaixo de 4 na avaliação de 2005 e alcançaram IDEB acima de 6 na avaliação de 2017 (24 escolas); 2) escolas que foram de um desempenho ruim para desempenho bom: de maneira semelhante à primeira categoria, foram selecionadas nesse grupo as escolas que tiveram IDEB abaixo de 5 na avaliação de 2005 e IDEB acima de 6 em 2017 (124 escolas). 
Uma Estratégia Regulatória Local para a Educação Básica no Rio de Janeiro

O outro lado do problema também demanda uma definição estreita (ou seja, qual critério serviria para identificar escolas recipientes de auxílio). Com isso em mente, foram definidas as escolas com desempenho abaixo do esperado como aquelas que cumpriam, concomitantemente, duas condições no município do Rio de Janeiro: 1) ocuparam o último quartil das notas do IDEB na avaliação de 2017 (159 escolas); e 2) ocuparam o último quartil da média das escolas nas últimas 4 avaliações do IDEB (2011, 2013, 2015 e 2017; 135 escolas). Qualquer um dos dois critérios, aplicados em isolamento, seria potencialmente enganoso. O primeiro porque, muito embora consiga capturar quais escolas estão com um desempenho pior neste momento, pode incluir escolas com um desempenho tradicionalmente excelente que, por conta de alguma situação meramente conjuntural, obtiveram um desempenho ruim no IDEB 2017. O segundo critério, por sua vez, poderia deixar de fora escolas que apresentaram alguma melhora no último IDEB, por mais que tenham tido dificuldades nas primeiras avaliações do recorte.

A aplicação conjunta dos critérios identificou 103 escolas que precisam ser auxiliadas por meio de políticas de Estado. Após a exclusão de duas escolas com dados incompletos ${ }^{10}$, restou um universo de 101 escolas nessa categoria (baixo desempenho, recipientes de auxílio).

O mapa abaixo representa, para cada escola com desempenho abaixo do esperado, a escola-referência mais próxima, permitindo uma visualização das posições geográficas:

Figura 2 - Escolas com desempenho abaixo do esperado e escolasreferência

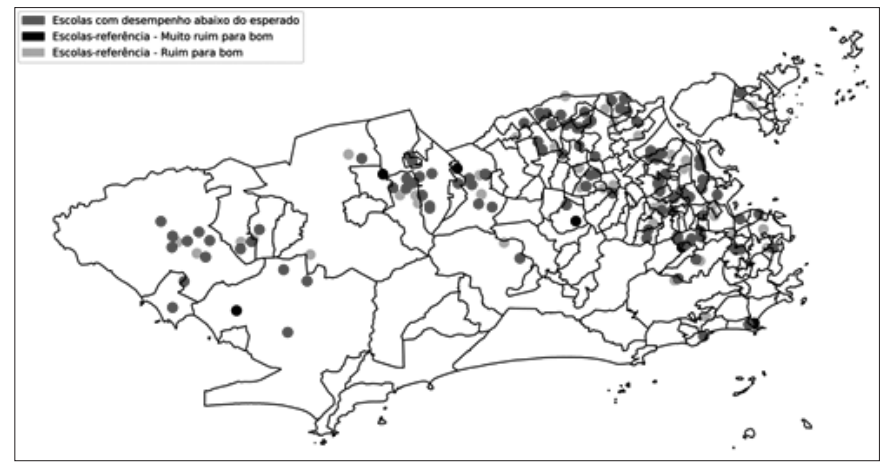

Fonte: Fonte: elaboração própria, a partir dos dados do IDEB (referentes ao período entre 2005 e 2017, INEP, 2020) e da prefeitura do Rio de Janeiro (PCRJ, 2019).

A figura 2 mostra a existência de escolas-referência ao lado de escolas com desempenho abaixo do esperado ${ }^{11}$. Nos casos mais emblemáticos para efeitos de comparação de desempenho, a escola-referência mais próxima está apenas a algumas centenas de metros da escola com desempenho abaixo do esperado (vide tabela 1). Em poucos casos, a distância entre a escola de risco e a escola-referência é grande (ver tabela 2), mas, ainda assim, essas distâncias podem não representar desafios comparativos relevantes para as duas escolas. 


\section{Tabela 1 - 10 menores distâncias}

\begin{tabular}{|c|c|c|}
\hline Escola com desempenho ruim & Escola-referência & $\begin{array}{l}\text { Distância } \\
\text { (metros) }\end{array}$ \\
\hline CIEP THOMAS JEFFERSON ${ }^{12}$ & ESCOLA MUNICIPAL LIMA BARRETO & 98.083 \\
\hline $\begin{array}{l}\text { ESCOLA MUNICIPAL ALBINO SOUZA } \\
\text { CRUZ }\end{array}$ & $\begin{array}{l}\text { ESCOLA MUNICIPAL EMA NEGRAO DE } \\
\text { LIMA }\end{array}$ & 103.77 \\
\hline ESCOLA MUNICIPAL JENNY GOMES & ESCOLA MUNICIPAL PEREIRA PASSOS & 246.9 \\
\hline $\begin{array}{c}\text { ESCOLA MUNICIPAL RUBENS BE- } \\
\text { RARDO }\end{array}$ & $\begin{array}{c}\text { ESCOLA MUNICIPAL PROFESSOR } \\
\text { AFONSO VARZEA }\end{array}$ & 253.62 \\
\hline CIEP DOUTOR NELSON HUNGRIA & $\begin{array}{l}\text { ESCOLA MUNICIPAL PROFESSOR JOAO } \\
\text { GUALBE... }\end{array}$ & 297.48 \\
\hline $\begin{array}{l}\text { ESCOLA MUNICIPAL ARAUJO PORTO } \\
\text { ALEGRE }\end{array}$ & ESCOLA MUNICIPAL MARIO FACCINI & 369.22 \\
\hline $\begin{array}{l}\text { ESCOLA MUNICIPAL PRESIDENTE JOAO } \\
\text { GOULART }\end{array}$ & ESCOLA MUNICIPAL PANAMA & 425.55 \\
\hline ESCOLA MUNICIPAL SOARES PEREIRA & $\begin{array}{l}\text { ESCOLA MUNICIPAL BARAO DE ITA- } \\
\text { CURUSSA }\end{array}$ & 428.06 \\
\hline CIEP CORONEL SARMENTO & ESCOLA MUNICIPAL EURICO VILLELA & 448.42 \\
\hline ESCOLA MUNICIPAL MEM DE AS & ESCOLA MUNICIPAL PEREIRA PASSOS & 469.14 \\
\hline
\end{tabular}

Tabela 2 - 10 maiores distâncias - elaboração própria, a partir dos dados do IDEB (referentes ao período entre 2005 e 2017, INEP, 2020) e da prefeitura do Rio de Janeiro (PCRJ, 2019)

\begin{tabular}{ccc}
\hline Escola com desempenho ruim & Escola-referência & $\begin{array}{c}\text { Distância } \\
\text { (metros }\end{array}$ \\
\hline CIEP DEPUTADO ULYSSES GUIMARAES & ESCOLA MUNICIPAL PROFESSORA & 5685.05 \\
ESCOLA MUNICIPAL PROFESSOR & MARIA SANT... \\
CASTILHO & ESCOLA MUNICIPAL PROFESSORA & 5333.84 \\
CIEP VILA KENNEDY & LEOCADIA T... & 3075.76 \\
ESCOLA MUNICIPAL JOANA ANGELICA & ESCOLA MUNICIPAL SAMUEL WAINER & 3058.39 \\
ESCOLA MUNICIPAL MOTORISTA PAS- & ESCOLA MUNICIPAL MANUEL DE & 3014.43 \\
CHOAL ANDRE & ABREU & 2993.62 \\
CIEP ANTONIO CANDEIA FILHO & ESCOLA MUNICIPAL ISAIAS ALVES & 2944.2 \\
ESCOLA MUNICIPAL JULIO CESARIO & ESCOLA MUNICIPAL PROFESSORA & MARIA SANT.. \\
DE MELO & ESCOLA MUNICIPAL MANUEL DE & 2943.04 \\
$\begin{array}{c}\text { ESCOLA MUNICIPAL ALZIRO ZARUR } \\
\text { ESCOLA MUNICIPAL PADRE JOSE }\end{array}$ & ABREU & \\
MAURICIO & ESCOLA MUNICIPAL RAYMUNDO & 2939.46 \\
ESCOLA MUNICIPAL GENERAL OSORIO & ESCOLA MUNICIPAL ISAIAS ALVES & 2789.42 \\
Fonte: Elaboração própria, a partir dos dados do IDEB (referentes ao período entre \\
2005 e 2017, INEP, 2020) e da prefeitura do Rio de Janeiro (PCRJ, 2019).
\end{tabular}


Uma Estratégia Regulatória Local para a Educação Básica no Rio de Janeiro

\section{Diferença de Desempenho Descorrelacionada de Fatores Socioeconômicos}

A motivação para selecionar os pares mais próximos é a presunção de que escolas geograficamente próximas são semelhantes com relação às variáveis top-down (ou seja, são recipientes similares dos programas de financiamento, do impacto futuro da base curricular, de um plano de carreira e de capacitação disponibilizado pelos entes federativos, entre outros). Além disso, IDH e PIB regionais, por exemplo, provavelmente se mantêm constantes para escolas vizinhas. Por outro lado, uma das variáveis mais importantes para o desempenho escolar, o nível socioeconômico dos alunos, pode variar entre escolas vizinhas, o que poderia explicar a diferença de resultado entre os pares de escolas identificados, a despeito da proximidade (em função da diferença entre os alunos). Será que as diferenças entre as escolas-referência e com desempenho abaixo do esperado podem ser explicadas por esse fator?

Dados do INSE (INEP, 2015b) ${ }^{13}$ foram usados para testar essa hipótese. Muito embora exista uma correlação entre o INSE e o IDEB absoluto para as 149 escolas avaliadas nessa fase do estudo ${ }^{14}$, o resultado não se replica para os pares de escolas. Quando se olha apenas para a distância entre o INSE da escola referência e da escola com desempenho abaixo do esperado e para a diferença de performance no IDEB entre o par de escolas, não é possível perceber qualquer correlação ${ }^{15}$, como pode ser observado no gráfico abaixo. Esse resultado pode ser interpretado da seguinte forma: muito embora certas regiões possuam uma concentração maior de escolas-referência e exista uma influência do INSE sobre o IDEB absoluto, a proximidade física dos pares de escola implica uma proximidade no quadro discente, de maneira que a variação entre os pares não pode ser explicada com referência ao nível socioeconômico dos alunos.

Figura 3 - Correlação entre as Diferenças do IDEB (2017) e do INSE (2015) para Cada Par de Escolas Mais Próximas ${ }^{16}$

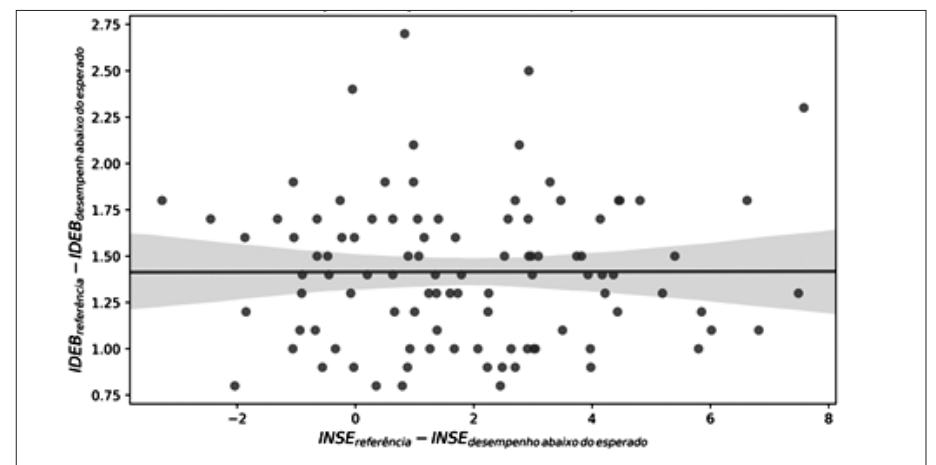

Fonte: Elaboração própria, a partir dos dados do IDEB (referentes a 2017; INEP, 2020) e do INSE (referente a 2015, INEP, 2015b). 
A partir dos dados acima, é possível ver que: (i) existe uma quantidade alta de escolas que precisam melhorar dentro do município do Rio de Janeiro; (ii) para a maioria dessas escolas, há uma escola-referência a uma distância razoável que pode servir de exemplo (para o possível desenho de medidas de gestão comparáveis e aplicáveis à escola com baixo desempenho). Conforme será exposto mais à frente, essas informações são extremamente relevantes para a regulação da educação municipal. Além disso, as variações socioeconômicas entre os alunos de cada um dos pares de escolas não foram capazes de explicar a diferença de desempenho, o que é sugestivo da efetividade das práticas de gestão locais implementadas nas escolas-referência ${ }^{17}$.

\section{Soluções Regulatórias e Problema de Principal/Agente: eficiểncia de instrumentos regulatórios locais}

A partir de 2007, o Ministério da Educação criou uma série de instrumentos com o objetivo de incentivar e auxiliar as políticas educacionais locais, tanto no nível dos Estados quanto dos municípios ${ }^{18}$. Em especial, uma série de ações foi desenvolvida sob o Plano de Desenvolvimento da Educação (PDE), objetivando o cumprimento do plano de metas Compromisso Todos pela Educação, incorporado ao Direito brasileiro por meio do Decreto no 6.094/2007, ao qual aderiram todos (ou pelo menos a maioria, vide Souza; Alcântara; Vasconcelos, 2014, p. 225) os municípios brasileiros. O principal modo descentralizado de incentivo ao desenvolvimento da educação no âmbito do PDE é a possibilidade da celebração de convênios entre a União e os Estados ou municípios envolvendo assistência técnica e financeira federal.

Para aderir ao PDE e se tornar elegível para as transferências financeiras e técnicas, cada município teve que analisar, à luz dos critérios estabelecidos pelo MEC, o desempenho de sua própria educação e, a partir desse diagnóstico, elaborar um Plano de Ações Articuladas (PAR), que serve de pauta para a celebração de eventuais convênios (Souza; Alcântara; Vasconcelos, 2014; Oliveira, 2014, p. 413). Na prática, o próprio sistema informático no qual os municípios devem cadastrar o instrumento de diagnóstico (Sistema Integrado de Planejamento, Orçamento e Finanças do Ministério da Educação - SIMEC) estabelece automaticamente as ações que devem ser implementadas pela secretaria de educação a partir das respostas fornecidas aos itens de avaliação ${ }^{19}$. Embora a adesão formal ao PAR tenha sido grande, em particular no Estado do Rio de Janeiro, o passo do diagnóstico é subvertido por incentivos perversos. Souza, Alcântara e Vasconcelos (2014), por exemplo, exploram a contradição entre o IDEB do Estado do Rio em 2011 - um dos 5 piores do Brasil - e a avaliação positiva que os municípios fizeram no diagnóstico do PAR.

As razões para essas discrepâncias podem estar atreladas à assimetria de informações combinada com interesses discrepantes, "[...] pois atribuindo pontuações mais elevadas estes [os municípios] se veem desobrigados da descrição das ações do PAR que deveriam ser 
Uma Estratégia Regulatória Local para a Educação Básica no Rio de Janeiro

adotadas”, além de evitarem “[...] maior regulação por parte das políticas do governo federal (já que, no caso das avaliações insatisfatórias, o Município tem que fazer frente às ações previstas no plano)" (p. 228). Assim, em um conjunto expressivo de vezes, o PAR parece não cumprir os seus objetivos descentralizadores ${ }^{20}$. Isso fica claro quando se nota que o acoplamento automático das ações ao diagnóstico faz com que os incentivos perversos descritos acima tenham consequências práticas.

Sob a perspectiva da análise econômica do direito, os problemas enfrentados na implementação do PAR parecem ser problemas do tipo principal-agente (Melo, 1996), em que os interesses da sociedade (principal) não são alcançados pelos responsáveis pela educação no município (secretários de educação municipais e, em última medida, diretores de escola).Como esse é um problema de informação, há necessidade de construção de instrumentos regulatórios locais para aumentar a transparência e accountability sobre as medidas necessárias para reparar o desempenho abaixo do satisfatório nas escolas identificadas ${ }^{21}$. E, por conta do problema principal-agente, agravado por possíveis custos adicionais associados a arranjos políticos (prefeitos e governadores podem ser de partidos políticos distintos entre eles, sendo que podem ainda ser opositores do governo federal), soluções federais provavelmente serão incapazes de efetivamente eliminar a diferença de desempenho. Parece, então, que a criação de uma governança regulatória local responsável por ações reparatórias é a forma mais eficiente de complementar a abordagem top-down, que efetivamente trouxe melhora de resultados nos últimos 15 anos. Para reverter o quadro de desempenho das escolas identificadas, é preciso olhar para uma série de fatores específicos de cada uma das unidades, se incluindo entre esses fatores a conduta dos diretores e a maneira como eles se relacionam com os professores (Bloom et al., 2015, Fundação Lemann; Itaú BBA, 2012); a conduta dos membros dos órgãos reguladores do Estado e seu contato com os diretores e, finalmente, a relação entre os pais dos alunos e a escola (Cunha et al. 2017; Coelho, 2008).

Políticas descentralizadoras têm sido adotadas em diversos países, com resultados positivos (vide Bloom et al., 2015). Exemplos paradigmáticos dessa tendência internacional pela valorização de estratégias bottom-up podem ser encontrados no Reino Unido, com o programa de academias - instituições de ensino básico completamente independentes das autoridades públicas (Eyles; Machin, 2019) - e em Portugal, onde a descentralização administrativa da rede pública é indicada como uma das razões para a melhora do desempenho do país no PISA (Liebowitz et al., 2018). Mesmo os EUA, que teve uma experiência descentralizadora controversa com o programa No Child Left Behind, aponta para a valorização de políticas locais (por exemplo, com a devolução aos Estados da regulação dos incentivos atrelados às avaliações de performance) como uma solução para os seus problemas (Sharp, 2016).

O maior caso de sucesso, amplamente utilizado como exemplo pela literatura, é o do município de Sobral, no Ceará, que passou de um IDEB de 4,0 nos anos iniciais do ensino fundamental em 2005 para nota 
9,1 em 2017 (Gramani, 2017; Menezes Filho, 2015; Inep, 2005a). Desde 2001 (Inep, 2005a), o município adota estratégias de gestão que buscam a erradicação do analfabetismo e da evasão escolar por meio da valorização do professor, do estabelecimento de gratificações e prêmios baseados no desempenho e da mensuração semestral dos resultados por uma comissão externa. O Estado do Ceará como um todo tem se destacado na educação básica por adotar estratégias como aquelas empregadas em Sobral, que enfatizam o caráter local da educação, muito embora ainda haja considerável espaço para melhoras (Gramani, 2017, Abrucio; Seggatto; Pereira, 2018).

No Rio de Janeiro, no entanto, não existe um instrumento regulatório que organize as ações reparadoras para escolas de baixo desempenho, trazendo transparência para as ações necessárias, metas de resultado de aprendizagem e mesmo incentivos ou sanções no caso do seu descumprimento. Há previsões importantes a respeito da educação básica na Lei Orgânica do Município, que, após repetir muitas das observações do texto constitucional, delega a regulamentação específica do tema para o Plano Municipal de Educação (PME) que, no entanto, não prevê um instrumento regulatório que seja capaz de orientar uma estratégia bottom-up. Em particular, cidades grandes, como é o caso do município do Rio de Janeiro, com um número bastante representativo de escolas ${ }^{22}$ precisam de uma governança regulatória que dê transparência para o desempenho de cada uma de suas escolas, bem como para o planejamento das ações individualizadas focadas nas escolas de baixo desempenho, o que só é possível a partir de avaliações empíricas tal como a elaborada na primeira seção deste artigo.

A última versão do PME do Rio de Janeiro foi aprovada com a lei $6.362 / 2018$. Estruturado por meio de conjuntos de metas e estratégias, o PME estabelece objetivos concretos com relação à ampliação do acesso e da qualidade da educação básica, incluindo notas alvo no IDEB e no PISA. No entanto, não há qualquer previsão quanto a ações reparatórias específicas $^{23}$, mais diretamente relacionadas às discrepâncias existentes entre as escolas da rede de ensino, embora existam estratégias locais para endereçar o problema de aprendizagem ${ }^{24}$. Também não existem indicações públicas a respeito de pesquisas no sentido de identificar as práticas que fazem com que certas escolas, diretores e professores se destaquem dos demais. A adoção de programas com base no PME do jeito que ele se encontra hoje pode não ser capaz de endereçar as dificuldades apresentadas pelas escolas de baixo desempenho, já que não permitem ações individualizadas típicas de uma abordagem bottomup para a implementação de política pública, nem tampouco estratégias que priorizem resultados mais efetivos na qualidade de aprendizagem, como o foco reparatório nos primeiros anos do ensino fundamental.

\section{Conclusão}

A análise dos dados do município do Rio de Janeiro demonstrou que existem escolas vizinhas com desempenhos díspares nos testes pa- 
Uma Estratégia Regulatória Local para a Educação Básica no Rio de Janeiro

dronizados. Há pares de escolas com desempenho abaixo do esperado e escolas-referência a menos de um quilômetro de distância uma da outra. Além disso, as diferenças socioeconômicas entre os alunos dessas escolas não são capazes de explicar a diferença em seu desempenho. No entanto, atualmente o conjunto de instrumentos regulatórios para endereçar medidas reparadoras é insuficiente, seja em razão dos problemas de principal e agente no acesso a instrumentos regulatórios federais ou mesmo em função da ausência de mecanismos regulatórios no município do Rio de Janeiro nessa direção.

De maneira geral, se percebe uma oportunidade para a implementação das políticas regulatórias descentralizadoras, que complementam estratégias top-down com estratégias bottom-up. Encontrar uma solução regulatória para a educação no município do Rio de Janeiro exige, portanto, uma consideração pormenorizada das características locais. O presente trabalho, por não envolver uma etapa empírica qualitativa, mas apenas quantitativa, ao identificar os pares de escolas que servem de confirmação para uma estratégia bottom-up, não é suficiente para encontrar uma solução. Seria uma contradição em termos pregar a importância das variáveis locais e delinear uma solução para o Rio de Janeiro baseada nas estratégias que funcionaram, por exemplo, no Reino Unido. Desde já, porém, é possível avançar em algumas conclusões.

A primeira é a que resulta da análise empírica realizada neste artigo: é necessário desenvolver instrumentos regulatórios bottom-up para o município do Rio de Janeiro. Na medida em que as variáveis locais não associadas a fatores socioeconômicos importam de maneira expressiva para o desempenho das escolas, parece óbvia a necessidade de desenvolver instrumentos regulatórios que exerçam influência local e sejam capazes de induzir o comportamento dos agentes. A relativa falta de produção regulatória no nível municipal - observada no caso do município do Rio de Janeiro - não é justificável ante à convergência de evidências empíricas e conceituais em torno da importância da gestão escolar. $\mathrm{O}$ aperfeiçoamento das evidências extraídas por meio de dados exige um aperfeiçoamento da gestão pública.

Além disso, em função do crescente resultado das políticas topdown, parece razoável que o foco dos instrumentos regulatórios locais deve se concentrar em reverter o quadro das escolas com pior desempenho. A ideia de que a educação pública deve ser oferecida com equidade é uma das ideias mais bem sedimentadas na literatura (Faria; Guimarães, 2015, Fundação Lemann; Itaú BBA, 2012, Comunitas; Juntos pelo Desenvolvimento Sustentável; FGV Ebape, 2017, Coelho, 2008). Razões de justiça social exigem que o foco dos esforços estatais seja despendido em prol das escolas em piores condições, em especial para os primeiros anos do ensino fundamental, tendo em vista o efeito que a aprendizagem nessa etapa terá nas etapas posteriores do ensino básico.

A existência de escolas-referência ao longo de todo o município do Rio de Janeiro mostra que é possível superar as limitações financeiras, administrativas e estruturais que afligem a educação pública e al- 
cançar resultados bons, o que sugere que estratégias bottom-up podem representar uma abordagem complementar de modo a entender como as práticas de gestão das escolas referência dentro da sua área geográfica pode encurtar o caminho para identificar a solução para reparar as escolas de baixo desempenho escolar. Ficou claro, também que essas soluções têm que ser desenhadas por agentes locais, em função do problema de principal agente que decorre de situações em que o auxílio depende de acesso a Governo Federal, o que, aliás, já existe (por meio do PAR) e que não foi capaz de reverter o quadro identificado para o município do Rio de Janeiro, que possui mais de 100 escolas em situação de reparo.

O exercício realizado neste artigo justifica a luta pela manutenção e pelo aprimoramento de instrumentos como o IDEB e o Censo Escolar, pois somente a partir de conjuntos confiáveis e consistentes de dados podemos avaliar adequadamente a educação, viabilizando, inclusive, o desenvolvimento de uma governança regulatória local capaz de criar instrumentos regulatórios adicionais de monitoramento (com propósitos e periodicidades distintas dos instrumentos federais de avaliação) e de reparação e auxílio escolar, proporcionando maior transparência e accountability para ações do governo visando corrigir os déficits educacionais dos municípios.

Por último, é importante ressaltar que o Rio de Janeiro (assim como o Brasil) já demonstrou por meio de exemplos concretos que tem experiências exitosas de educação (a escola municipal com maior IDEB no ensino fundamental tem nota 8,0 no índice). A dificuldade é estender a escolas com baixo desempenho esse conhecimento, gerando escala no aprendizado de técnicas de gestão que possuem impacto positivo no IDEB. Novos instrumentos regulatórios (como, por exemplo, o sugerido neste artigo a partir da identificação de abordagens inspiradas no uso de escolas referências em par de escolas) permitiriam diferentes soluções para diferentes problemas a partir de uma única abordagem que identifica possíveis práticas aplicáveis em cidades com perfil semelhante ao Rio de Janeiro, em que houve uma melhora nos indicadores, porém com impactos diferentes em escolas no município, demandando ações reparadoras individualizadas para um número relevante de unidades da rede.

Recebido em 24 de junho de 2019 Aprovado em 29 de junho de 2020

\section{Notas}

1 Agradecemos a Fernanda Scovino pelo auxílio com o tratamento de dados e as análises estatísticas realizadas.

2 Embora, recentemente, tenha surgido um movimento ganhando força para a desvinculação do orçamento no nível federal, incluindo os gastos com saúde e educação. Disponível em: <https://brasil.elpais.com/brasil/2019/01/02/ politica/1546459750_824035.html>. Acesso em: 20 jun. 2019. 
Uma Estratégia Regulatória Local para a Educação Básica no Rio de Janeiro

3 Neste artigo, adota-se a tipologia nos termos e com o sentido indicado por Pülzl; Treib (2007). Segundo os autores, estratégias top-down “[...] colocam sua ênfase principal na habilidade dos tomadores de decisões de produzir objetivos políticos inequívocos e em controlar a etapa de implementação" (p. 90, tradução nossa). Em contraste, estratégias bottom-up “[...] veem os burocratas locais como os principais atores na entrega de políticas e concebem da implementação como processos de negociação dentro de redes de implementadores" (Püzl; Treib, 2007, p. 90, tradução nossa). O consenso identificado pelos autores a respeito da distinção é de que se trata de um contínuo: políticas podem ser mais ou menos top-down ou bottom-up, a depender do grau de autonomia que elas dão aos burocratas locais (p. 100, tradução nossa).

4 O IDEB é uma métrica padronizada de avaliação da educação que leva em consideração dois fatores: 1) o desempenho acadêmico dos alunos na Prova Brasil e 2) as taxas de aprovação de cada escola. Segundo a apresentação do documento que introduziu o instrumento, assinado por Oroslinda Goulart, essa opção está justificada: “[...] [s]eu pressuposto é a evidente complementariedade entre ambos, lembrando que, mesmo que os alunos atinjam elevadas pontuações nos exames padronizados, um sistema educacional que reprova sistematicamente seus estudantes, provocando o abandono de um número significativo deles, sem que completem a educação básica, não é desejável" (Fernandes, 2007, p. 5). Uma das características marcantes do IDEB é a possibilidade de que duas escolas alcancem a mesma nota por meio de diferentes combinações entre nota na Prova Brasil e taxa de aprovação.

5 Em 2007, o IDEB nacional era de 4,0, tendo alcançado nota 5,5 em 2017.

6 O INSE (Indicador de Nível Socioeconômico da Educação Básica) é uma métrica que tem como objetivo capturar as diferenças socioeconômicas dentre os alunos das escolas da educação básica pública. Vide Inep (2015b).

7 Nesse sentido: “[...] a alfabetização é a base de uma escolarização bem-sucedida e influi na redução da distorção idade-série e dos índices de abandono [...]" (Inep, 2005a, p. 15).

8 O modesto aumento do IDEB do Ensino Médio da rede pública nacional de 3,1 em 2007 para 3,5 em 2017 é consistente com nossa hipótese. Afinal, a previsão é justamente de que aumentos de rendimento em séries iniciais possibilitam aumentos no rendimento das séries finais.

9 As análises que se seguem levam em consideração o IDEB da escola como variável dependente. Uma crítica levantada contra essa estratégia aponta para a possibilidade de as escolas poderem chegar a um mesmo IDEB através de resultados diferentes. Dado que esse é o caso, pelo menos alguns dos pares que identificamos poderiam representar um desempenho acadêmico similar, com diferenças apenas nas taxas de aprovação. A crítica é válida. Análises levando em consideração diretamente a nota da escola no SAEB, porém, mostram que permanecem existindo pares de escolas vizinhas com desempenhos discrepantes. Há sobreposição entre os pares identificados usando o IDEB ou o SAEB, mas também há divergência. Isso, porém, é menos importante do que a persistência das hipóteses do trabalho, já que não há a pretensão, ao menos ainda, de indicar exatamente quais pares específicos de escolas devem ser priorizados por políticas públicas, mas somente mostrar a conveniência de uma perspectiva bottom-up.

10 São as escolas municipais Mario Lago e Raphael Almeida Magalhães. 
11 O Estado do Ceará, segundo Abrucio; Seggatto; Pereira (2018) estabeleceu uma competição administrada entre os municípios. Segundo os autores “[...] [t]ratase de um modelo em que o governo estabelece mecanismos controlados de competição, visando a aumentar o desempenho de órgãos serviços públicos e entes territoriais. Nesta situação, são construídos indicadores para mensuração dos resultados, e geralmente são distribuídos recursos (financeiros e gerenciais) conforme a performance dos participantes. Procura-se, também, criar estímulos para motivar os que têm pior desempenho e mecanismos de apoio a estes, incluindo muitas vezes as necessidades de os 'melhores' ajudarem os 'piores'” (p. 39). É exatamente esse o espírito da análise de pares de escolas: uma vez identificados, podemos fazer com que as melhores ajudem as piores.

12 Boa parte dos CIEPs, programa historicamente associado ao governo do Estado do Rio de Janeiro, foi municipalizada ao longo dos últimos anos. Vide Cavaliere; Coelho (2013), para uma retrospectiva.

13 Não encontramos os dados do INSE referentes à escola municipal Mario Faccini, razão pela qual a excluímos da análise.

$14 \mathrm{R}=0,43, \mathrm{p}<0,001$.

$15 \mathrm{R}=0,002, \mathrm{p}=0,98$.

16 No eixo X, estão representadas as diferenças de nível socioeconômico entre cada um dos membros dos pares de escolas identificados. No eixo Y, está a diferença de IDEB entre a escola de referência e a escola com desempenho abaixo do esperado. A ausência de um padrão indica que não podemos reduzir as diferenças observadas no IDEB às condições socioeconômicas dos alunos das escolas.

17 Outra possibilidade que merece ser explorada é a de que as escolas tenham desempenhos discrepantes conforme a sua inserção em comunidades de risco. Para verificar se a diferença entre os pares de escola poderia ser explicada por esse fator, foram utilizados os dados da prefeitura do Rio de Janeiro sobre os limites de favelas (disponível em: <http://www.data.rio/datasets/limitefavelas $>$. Acesso em: 12 jun. 2019). Apenas 3 das 101 escolas com desempenho abaixo do esperado se encontram dentro dos limites descritos pela prefeitura. Mesmo quando ampliamos a área das favelas em 20\%, apenas 19 escolas com desempenho abaixo do esperado estão dentro do perímetro estabelecido. Portanto, mesmo que essas unidades fossem eliminadas da nossa comparação, restariam 82 pares de escolas referência e escolas com desempenho abaixo do esperado cuja diferença não poderia ser reduzida a fatores socioeconômicos de alguma natureza. Optou-se por manter essas 19 escolas na análise principal por alguns motivos. Em primeiro lugar, não há nenhum bom motivo regulatório para excluir as escolas dentro de favelas. Se há vontade de promover a equidade, é especialmente importante olhar para as escolas que mais precisam de ajuda. Assim, ainda que o interesse acadêmico justifique a análise em separado, a motivação prática recomenda a reinserção das escolas no estudo. Em segundo lugar, seria necessário explorar quais fatores territoriais dissociados das condições socioeconômicas do corpo discente poderiam explicar de maneira adequada eventuais diferenças entre escolas que se encontram dentro e fora dos limites das favelas. Muito embora essa diferença tenha sido percebida ao se controlar para os efeitos do INSE, optou-se, por entender essa questão como tangencial ao argumento principal, por não explorar hipóteses explicativas nessa ocasião. 
Uma Estratégia Regulatória Local para a Educação Básica no Rio de Janeiro

18 Aqui, é importante notar uma distinção entre estratégias bottom-up e estratégias locais. Ao determinar de maneira central quais são os tipos de problemas e soluções que podem ser encontrados pelos municípios, entendemos que os PAR são um instrumento local, porém top-down. O que importa para definir se uma política é bottom-up ou top-downé a centralização da tomada de decisão e a relativa falta de flexibilidade encontrada pelos implementadores na ponta, e não seu âmbito de aplicação.

19 Para um detalhamento das ações, ver Ministério da Educação, 2011a; para um passo a passo sobre o procedimento de geração do PAR, Ministério da Educação, 2011b.

20 Para uma análise detida do caso do município de Campos dos Goytacazes, ver Paula (2017).

21 No Brasil, o PL 7.420/2006, intitulado Lei de Responsabilidade Educacional, tentou regular os incentivos locais envolvidos no ensino de maneira ampla. O projeto previa a avaliação de desempenho de alunos com o estabelecimento de que os resultados deveriam ser sempre melhores do que aqueles ocorridos no ano anterior; metas de melhora progressiva com relação às taxas de repetência e evasão escolar e a obrigatoriedade da confecção de um plano de educação. O não cumprimento dessas medidas, por sua vez, afetaria as transferências voluntárias da União e poderia acarretar, inclusive, em crimes de responsabilidade ou atos de improbidade administrativa. O projeto de Lei de Responsabilidade Educacional de fato manipula variáveis que importam para a solução do problema. Planos de educação diminuem a assimetria de informação nas relações entre a sociedade e os gestores educacionais, enquanto o estabelecimento de standards de performance em termos do desempenho dos alunos em prova e das taxas de evasão escolar e de repetência diminuem a assimetria de informação com relação aos resultados do processo educacional. De toda forma, o projeto segue em tramitação, não tendo sido incorporado ao ordenamento jurídico brasileiro.

22 Apenas para contextualizar, pode ser que a estratégia de Sobral não seja replicável no município do Rio de Janeiro, já que se trata de ordens de grandeza totalmente diferentes. Sobral possui, segundo o Censo Escolar de 2018, 14.543 estudantes matriculados nos anos iniciais do ensino fundamental, contra 404.008 estudantes na cidade do Rio de Janeiro. As informações foram extraídas da plataforma Qedu (www.qedu.org.br), alimentada com dados do Censo Escolar de 2018. As condições sociodemográficas e culturais das duas cidades também são muito diferentes.

23 O PL 7.420/2006 criava um modelo de incentivo baseado em sanção, reconhecendo a importância do caráter local dos problemas envolvendo a educação. $\mathrm{O}$ não cumprimento das metas estabelecidas por escolas geraria uma obrigação por parte das Unidades da Federação de “[...] desenvolver ações específicas, com a necessária destinação de recursos financeiros, compatível com as necessidades de superação das causas que estejam determinando as insuficiências observadas em seus sistemas e redes públicas de ensino" (art. $2^{\circ}, \S 4^{\circ}$ ). A compreensão de que 1) qualquer situação de desempenho abaixo do esperado precisa ser enfrentada e 2) este enfrentamento deve se dar no nível local é fundamental para entender o perfil do tipo de instrumento regulatório que teria efeito no desenvolvimento das escolas com baixo desempenho, embora o modelo baseado em sanções seja imperfeito para gerar resultados positivos, já que é necessária criar uma estrutura regulatória mínima prévia para avaliar a 
responsabilidade do gestor, o que não existe no maior número de municípios que hoje existem no Brasil.

24 Como, por exemplo, a estratégia 19.5, que pretende: “[...] estimular a participação e a consulta de profissionais da educação, alunos e seus familiares na formulação dos projetos político-pedagógicos, currículos escolares, planos de gestão escolar e regimentos escolares, assegurando a participação dos pais na avaliação de docentes e gestores escolares".

\section{Referências}

ABRUCIO, Fernando Luiz; SEGGATTO, Catarina Ianni; PEREIRA, Maria Cecília Gomes. Regime de Colaboração no Ceará: funcionamento, causas do sucesso e alternativas de disseminação do modelo. São Paulo: Instituto Natura, 2018. Disponível em: <http://www.institutonatura.org.br/wp-content/uploads/2018/10/ Pesquisa-FGV__PAIC.pdf>. Acesso em: 12 jun. 2019.

ALVES, Maria Teresa; SOARES, José Francisco. As Pesquisas Sobre o Efeito das Escolas: contribuições metodológicas para a sociologia da educação. Sociedade e Estado, Brasília, v. 22, n. 2, p. 435-473, maio/ago. 2007a.

ALVES, Maria Teresa; SOARES, José Francisco. Contexto Escolar e Indicadores Educacionais: condições desiguais para a efetivação de uma política de avaliação educacional. Educação e Pesquisa, São Paulo, v. 39, n. 1, p. 177-194, jan./ mar. 2013b.

ANDRADE, Josemberg Moura; LAROS, Jacob Arie. Fatores Associados ao Desempenho Escolar: estudo multinível com dados do SAEB/2001. Psicologia: teoria e pesquisa, Brasília, v. 23, n. 1, p. 33-41, jan./mar. 2007.

BLOOM, Nicholas; LEMOS, Renata; SADUN, Raffaella; VAN REENEN, John. Does Management Matter in Schools? The Economic Journal, n. 125, p. 647-674, maio 2015.

CAVALIERE, Ana Maria; COELHO, Lígia Martha. Trajetória dos CIEPs do Rio de Janeiro: municipalização e novas configurações. Educação em Foco, Juiz de Fora, v. 18, n. 2, jul./out. 2013, p. 213-242.

COELHO, Maria Inês de Matos. Vinte Anos de Avaliação da Educação Básica no Brasil: aprendizagens e desafios. Ensaio: avaliação e políticas públicas em educação, Rio de Janeiro, v. 16, n. 59, p. 229-258, abr./jun. 2008.

COMUNITAS; JUNTOS PELO DESENVOLVIMENTO SUSTENTÁVEL; FGV EBAPE. Como Melhorar o Ideb: experiências que funcionam. 2017. Disponível em: <https://wiki.redejuntos.org.br/system/files/2017-10/cartilhaeducacaoweb-2_0.pdf>. Acesso em: 15 maio 2019.

CUNHA, Nina; LICHAND, Guilherme; MADEIRA, Ricardo; BETTINGER, Eric. What Is It About Communicating with Parents? Stanford: Stanford, 2017. Disponível em: <https://cepa.stanford.edu/sites/default/files/cunha_cover_paperl.pdf>. Acesso em: 02 mai. 2019.

EYLES, Andrew; MACHIN, Stephen. The Introduction of Academy Schools to England's Education. Journal of the European Economic Association, v. 17, n. 4, ago. 2019, p. 1107-1146.

FARIA, Ernesto Martins; GUIMARÃES, Raquel Rangel de Meireles. Excelência com Equidade: fatores escolares para o sucesso educacional em circunstâncias desfavoráveis. Estudos em Avaliação Educacional, v. 26, n. 61, jan./abr. 2015, p. $192-215$ 
Uma Estratégia Regulatória Local para a Educação Básica no Rio de Janeiro

FERNANDES, Reynaldo. Índice de Desenvolvimento da Educação Básica (Ideb). Brasília: Instituto Nacional de Estudos e Pesquisas Educacionais Anísio Teixeira, 2007.

FUNDAÇÃO LEMANN; ITAÚ BBA. Excelência com Equidade: as escolas que oferecem um ensino de qualidade aos alunos com baixo nível socioeconômico, 2012. Disponível em: <https://fundacaolemann.org.br/materiais/excelenciacom-equidade>. Acesso em: 02 mai. 2019.

GRAMANI, Maria Cristina. Análise dos Determinantes de Eficiência Educacional do Estado do Ceará. Ensaio: avaliação e políticas públicas em educação, Rio de Janeiro, v. 25, n. 95, abr./jun. 2017, p. 507-526.

INEP. Vencendo o Desafio da Aprendizagem nas Séries Iniciais: a experiência de Sobral/CE. Brasília: INEP, 2005a. Disponível em: <http://portal.inep.gov.br/ documents/186968/488938/Vencendo+o+desafio+da+aprendizagem+nas+s\%C 3\%A9ries+iniciais+a+experi\%C3\%AAncia+de+Sobral-CE/a7de6174-3f52-49feb81c-9f40372761a3?version=1.0>. Acesso em: 08 maio 2019.

INEP. Nota Técnica - Indicador de Nível Socioeconômico das Escolas de Educação Básica (Inse). Brasília: INEP, 2015b. Disponível em: <http://download.inep. gov.br/informacoes_estatisticas/indicadores_educacionais/2015/nota_tecnica/nota_tecnica_inep_inse_2015.pdf >. Acesso em: 11 jun. 2019.

INEP. IDEB - Resultados. Brasília: INEP, 2020. Disponível em: <http://portal. inep.gov.br/web/guest/educacao-basica/ideb/resultados>. Acesso em 13 jul. 2020.

LIEBOWITZ, David; GONZÁLEZ, Pablo; HOOGE, Edith; LIMA, Gonçalo. OECD Reviews of School Resources: Portugal, 2018. OECD Publishing: Paris, 2018.

MARTINS, Paulo de Sena. Constituinte, Financiamento e Direito à Educação: a voz dos protagonistas. Educação \& Sociedade, Campinas, v. 39, n. 145, out./dez. 2018, p. 823-845.

MELO, Marcos André. Governance e Reforma do Estado: o paradigma agente x principal. Revista do Serviço Público, Brasília, v. 120, n. 1, jan./abr. 1996, p. 67-82.

MENEZES FILHO, Naercio. Como Melhor a Educação no Brasil? Revista Interesse Nacional, v. 31, out./dez. 2015. Disponível em: <http://interessenacional. com.br/2015/10/05/como-melhorar-a-educacao-no-brasil/>. Acesso em: 08 maio 2019 .

MINISTÉRIO DA EDUCAÇÃO. Plano de Ações Articuladas - PAR 2011-2014. Guia Prático deAções para Municípios. Brasília:MEC,2011a.Disponívelem:<http://portal.mec.gov.br/index.php?option=com_docman\&view=download\&alias $=9435$ guia-pratico-acoes-par2011-2014-nov\&category_slug=novembro-2011pdf\&Itemid=30192> . Acesso em: 17 jun. 2019.

MINISTÉRIO DA EDUCAÇÃO. Orientações para Elaboração do Plano de Ações Articuladas (PAR) dos Municípios (2011-2014). Brasília: MEC, 2011b. Disponível em: <http://portal.mec.gov.br/index.php?option=com docman\&view=download\&alias $=9400$ - manualelaboracao-par-municipal1114-pdf\&category_slug=novembro-2011-pdf\&Itemid=30192>. Acesso em: 17 jun. 2019.

OLIVEIRA, Adailda Gomes de. Políticas Educacionais e a Qualidade da Educação nos Municípios Fluminenses: alguns recortes. Ensaio: Avaliação e Políticas Públicas em Educação, Rio de Janeiro, v. 22, n. 83, p. 411-442, abr./jun. 2014.

PAULA, Otávio Cordeiro de. O Plano de Desenvolvimento da Escola como Ins- 
trumento de Gestão das Escolas Públicas Municipais de Campos dos Goytacazes-RJ. 2017. 193f. Tese (Doutorado em Educação) - Departamento de Educação, PUC-Rio, Rio de Janeiro, 2017.

PREFEITURA DA CIDADE DO RIO DE JANEIRO - PCRJ. Dados sobre Escolas Municipais. Rio de Janeiro. Disponível em: <http://hub.arcgis.com/datasets/ PCRJ::escolas-municipais >. Acesso em: 13 maio 2019.

PÜLZL, Helga; TREIB, Oliver. Implementing Public Policy. 2007. In: FISCHER, Frank; MILLER, Gerald, SIDNEY, Mara. (Org.). Handbook of Public Policy Analysis: theory, politics and methods. Boca Raton: CRC Press, 2007. P. 89-107.

SASSAKI, Alex Hayato; DI PETRA, Giovanni; MENEZES FILHO, Naercio; KOMATSU, Bruno. Por Que o Brasil Vai Mal no PISA? uma análise dos determinantes do desempenho no exame. Insper. PolicyPaper, n. 31, jun. 2018. Disponível em: <https://www.insper.edu.br/wp-content/uploads/2018/08/Por-que-Brasilvai-mal-PISA-Analise-Determinantes-Desempenho.pdf $>$. Acesso em: 16 maio 2019.

SCHREINER, Clara. International Case Studies of Smart Cities: Rio de Janeiro, Brazil. Inter-American Development Bank, Discussion Paper n. IDB-DP-447, 2016. Disponível em: <https://publications.iadb.org/en/international-casestudies-smart-cities-rio-de-janeiro-brazil>. Acesso em: 16 maio 2019.

SHARP, Laurie. ESEA Reauthorization: an overview of the every student succeeds act. Texas Journal of Literacy Education, v. 4, i. 1, summer 2016, p. 10-16.

SOUZA, Donaldo Bello de; ALCÂNTARA, Alzira Batalha; VASCONCELOS, Maria Celi Chaves. Diagnósticos Locais do Plano de Ações Articuladas: uma análise de sua confiabilidade. Educação, v. 37, n. 2, p. 218-231, maio/ago. 2014.

TODOS PELA EDUCAÇÃO. Anuário Brasileiro da Educação Básica, 2018. São Paulo: Moderna, 2018. Disponível em: <https://todospelaeducacao.org. br/_uploads/20180824-Anuario_Educacao_2018_atualizado_WEB.pdf?utm_ source $=$ conteudoSite $>$. Acesso em: 30 maio 2019.

Carlos Emmanuel Joppert Ragazzo é professor do Corpo Permanente do Curso de Pós Graduação Stricto Senso da FGV Direito Rio (Mestrado em Direito da Regulação). Doutor e Mestre em Direito pela Universidade do Estado do Rio de Janeiro (UERJ).

ORCID: http://orcid.org/0000-0003-4049-7691

E-mail: cjragazzo@gmail.com

Guilherme da Franca Couto Fernandes de Almeida é Coordenador do Supremo em Números, FGV Direito Rio. Mestre e doutorando em Teoria do Estado e Direito Constitucional pela Pontifícia Universidade Católica do Rio de Janeiro (PUC-Rio).

ORCID: http://orcid.org/0000-0002-9134-9843

E-mail: guilherme.almeida@fgv.br

Editora-responsável: Beatriz Vargas Dorneles

Este é um artigo de acesso aberto distribuído sob os termos de uma Licença Creative Commons Atribuição 4.0 Internacional. Disponível em: <http:// creativecommons.org/licenses/by/4.0>. 